\title{
Implementation of crowding solutions from the American College of Emergency Physicians Task Force Report on Boarding
}

\author{
Daniel A. Handel • Adit A. Ginde • Ali S. Raja • \\ John Rogers • Ashley F. Sullivan • Janice A. Espinola • \\ Carlos A. Camargo Jr.
}

Received: 3 June 2010 /Accepted: 29 June 2010 /Published online: 21 August 2010

(C) Springer-Verlag London Ltd 2010

\begin{abstract}
Study Objective We sought to measure the self-reported implementation of the crowding solutions outlined in the 2008 American College of Emergency Physicians (ACEP) Boarding Task Force report "Emergency Department Crowding: High-Impact Solutions." We also tested the hypothesis that the self-reported crowding of emergency departments (EDs) was positively associated with the implementation of these solutions.

Methods In early 2009, we mailed a survey to all medical or nursing directors from EDs in four US states
\end{abstract}

The views expressed in this paper are those of the author(s) and not those of the editors, editorial board or publisher.

D. A. Handel $(\bowtie)$

Center for Policy and Research,

Department of Emergency Medicine,

Oregon Health \& Science University,

Portland, OR, USA

e-mail: handeld@ohsu.edu

\section{A. A. Ginde}

Department of Emergency Medicine,

University of Colorado Denver School of Medicine,

Aurora, CO, USA

A. S. Raja

Department of Emergency Medicine,

Brigham and Women's Hospital,

Boston, MA, USA

\section{J. Rogers}

Department of Emergency Medicine, Monroe County Hospital, Forsyth, GA, USA

A. F. Sullivan · J. A. Espinola $\cdot$ C. A. Camargo Jr.

Department of Emergency Medicine,

Massachusetts General Hospital,

Boston, MA, USA asking for information regarding their EDs in 2008. Geographic information about the EDs was included in the analysis, along with survey responses about their ED capacity status and implementation of specific ACEP crowding solutions.

Results A total of 284 of 351 EDs responded (81\%). The majority of EDs were in urban areas (56\%), non-teaching hospitals (93\%), and not critical access hospitals (76\%). The percentage of EDs "over capacity" ranged from 10 $49 \%$ in each state. The mean number of crowding solutions used in EDs that were at or over capacity ranged from 3.6-4.6 in each state. EDs with visit volumes greater than or equal to three patients/hour were more likely to be over capacity than at capacity or at a good balance ( $46 \%$ vs. $31 \%$ and $15 \%$, respectively). In terms of the use of high-impact crowding solutions, hospitals over capacity were more likely to utilize inpatient full capacity protocols $(40 \%$ vs. $25 \%$ and $25 \%$ ) but not inpatient discharge coordination (29\% vs. $27 \%$ and $34 \%$ ) or surgical schedule smoothing (31\% vs. $28 \%$ and $32 \%$ ). Hospitals over capacity were also more likely to have fast track units (44\% vs. $32 \%$ and $16 \%$ ) and physicians at triage ( $48 \%$ vs. $29 \%$ and $17 \%$ ).

Conclusion Less than half of EDs in each state reported operation above capacity. Implementation of some crowding solutions was more common in the abovecapacity EDs, although these solutions were not consistently used across geographic locations and hospitals. Given that the majority of EDs were not over capacity, the implementation of these solutions does not seem to be universally necessary.

Keywords Emergency department crowding · ACEP. Crowding solutions 
In July 2008, the American College of Emergency Physicians (ACEP) published their Task Force Report on Boarding, "Emergency Department Crowding: HighImpact Solutions" [1]. The Report was novel in that its focus was on solutions to Emergency Department (ED) crowding, an issue that continues to worsen [2] as patients face ever increasing wait times [3].

After defining ED crowding and its adverse consequences, the report explored both internal ED process improvement actions as well as hospital-wide solutions. It summarized that three hospital-wide solutions would have a high impact on improving the flow of patients through the ED: (1) move emergency patients who have been admitted to the hospital out of the ED to inpatient areas, such as hallways, conference rooms, and solaria; (2) coordinate the discharge of hospital inpatients before noon, as timely discharge of patients can significantly improve the flow of patients through the ED by making more inpatient beds available to emergency patients [4]; (3) coordinate the scheduling of elective patients and surgical patients. Other solutions, both within the ED and throughout the hospital, were also recommended: (1) bedside registration, (2) fast track units, (3) observation units, (4) physician triage, and (5) elective surgery cancellation [1].

This study is the first to look at the implementation of these ED crowding solutions in multiple states. To date, no data exist on which solutions have been implemented, especially in non-academic EDs with lower volumes (e.g., $<8,760$ visits/year or $<1$ patient/hour). The objectives of this cross-sectional multi-state study were to measure the implementation of crowding solutions in EDs with varying geography and annual visit volumes and to determine if there is an association between crowding and the use of crowding solutions.

\section{Methods}

\section{Study design}

We mailed surveys to directors of all EDs in Colorado, Georgia, Massachusetts, and Oregon between January and April of 2009. The study was approved by each state investigator's Institutional Review Board with a waiver of written informed consent.

We used the 2007 version of the National Emergency Department Inventories (NEDI)-USA database to obtain a comprehensive list of all nonfederal US hospitals with EDs in the four included states $(\mathrm{n}=351)$. Methods for derivation of NEDI-USA have been previously described [5]. We compiled the inventory through original data collection and integration of information from a variety of sources (e.g., Verispan Hospital Market Profiling Solution, American
Hospital Association Annual Survey Database, Flex Monitoring Team, and Association of American Medical Colleges). EDs were defined as emergency care facilities open $24 \mathrm{~h}$ per day, 7 days per week, and available for use by the general public. We excluded federal hospitals (e.g., Veterans Affairs and Indian Health Service hospitals) and college infirmaries.

\section{Study protocol}

We mailed the survey to ED directors three times over a 2 -month period. Non-responders and those with partial or incomplete responses were contacted by telephone and/or e-mail for completion. Each state had an investigator responsible for survey distribution and collection. Either the medical director and/or the nursing director were eligible to complete the survey. We left it to the directors' discretion from what personnel within their department they sought input to the survey questions. When contacted by phone, they were given the option to complete the survey over the phone or have an additional copy of the survey faxed to them. Once received by the EMNet Coordinating Center, the surveys were checked by trained research assistants for omissions and errors. State site investigators and research assistants followed up with directors to complete missing data.

\section{Measurements}

We classified ED location as urban and rural (adjacent to urban or not adjacent to urban) using the county-based 2003 Urban Influence Codes (www.usda.gov). EDs were categorized by their self-reported capacity status (under capacity, good balance, at capacity, and over capacity). This categorization of capacity has been used in prior national ED studies [6]. Other ED characteristics included in the survey were the average number of ED patients seen per hour (calculated from annual visit volume), hospital admission rate, and percent of uninsured patients. Physician staffing characteristics included total emergency physician full-time equivalents and proportion of Emergency Medicine Board Certified/Board Eligible (EM BC/ $\mathrm{BE})$ physicians by the American Board of Emergency Medicine, American Osteopathic Board of Emergency Medicine, or the American Board of Pediatrics (Pediatric EM).

Questions on crowding were part of a larger threepage survey (Appendix). These questions were based on the ACEP Task Force's recommended solutions. ${ }^{1}$ We requested input from the Board of Directors from all four states' ACEP state chapters and Steering Committee members of EMNet (www.emnet-usa.org) with regards to the content of the survey. To compare the level of 
crowding solutions implemented by hospitals, a composite score was created for questions E6a-d and E7a-e. One point was assigned for every solution utilized partially or completely. Possible scores ranged from $0-9$, with 9 being the most solutions implemented.

Respondents were asked to provide estimates for either the calendar or fiscal year 2008, whichever was available at the time of receipt of the survey. All respondents were assured that their responses would be anonymous, and individual ED identifiers were removed prior to analysis. They were asked to provide estimates for all patients seen during this time period. "Partial/In Progress" responses were analyzed as affirmative implementations.

\section{Data analysis}

The primary unit of analysis for the survey was the ED. We performed statistical analyses using Stata 10.1 (StataCorp, College Station, TX) and summarized data using basic descriptive statistics such as proportions and mean composite scores of the number of high impact solutions implemented (with 95\% confidence intervals). We performed the univariable analyses using chi-square and Fisher's exact tests to compare differences in use of crowding solutions by ED characteristics and capacity. All $P$ values were two-tailed, with $P<0.05$ considered statistically significant. We then performed multivariable linear regression to evaluate independent predictors of at or over capacity EDs with results reported as beta coefficients with $95 \%$ confidence intervals (CIs). ED characteristics obtained from the survey and NEDI-USA, including location (state and urban vs. rural), teaching vs. non-teaching, critical access hospital vs. not, volume, admission rate, and percentage of uninsured patients, were included a priori in the multivariable model.

\section{Results}

A total of 284 of 351 EDs responded to the survey $(81 \%)$. The majority of these were in urban areas (56\%), non-teaching hospitals (93\%), and not critical access hospitals $(76 \%)$. From a crowding perspective, those with visit volumes $\geq 3$ patients/hour were more likely to be at or over capacity than those with a lower hourly volume (Table 1). Overall, only a minority (26\%) of EDs stated that they were over capacity, regardless of whether they were urban vs. rural, teaching vs. non-teaching, or had high proportions of admitted or uninsured patients. In each of these categories, the percentage of EDs operating over capacity ranged from $15-35 \%$ (Table 1 ). There was also variation between states in terms of the percentage of
EDs operating over capacity, with the lowest in Colorado at $10 \%$ and the highest in Massachusetts at $49 \%$.

In terms of the use of high-impact crowding solutions, hospitals over capacity were more likely to utilize inpatient full capacity protocols, but not inpatient discharge coordination and surgical schedule smoothing (Table 2). They were also more likely to have fast track units and a physician at triage. Those operating at a good balance were more likely to have bedside registration and/ or had eliminated triage. There was also a higher median percentage of patients who left before being seen at EDs over capacity. The number of High Impact Solutions by crowding status can be seen in Table 3, in which those EDs that were at or over capacity had implemented a greater number of high-impact solutions.

No statistically significant associations were found in the composite score of any crowding solution for EDs at or over capacity in terms of rural vs. urban, teaching hospitals, critical access hospitals, or insurance status. However, composite scores increased significantly with higher visit volumes and admission rates (Table 4). In a multivariable linear regression analysis, only higher visit volumes were found to be significantly associated with higher composite scores for any crowding solution (Table 5).

\section{Discussion}

Despite widespread concerns about ED crowding, our results demonstrated that it is not a pervasive phenomenon. In particular, hourly patient volume seemed to correlate with self-perceived capacity and the composite score of crowding solutions implemented. Interestingly, perceived crowding varied by state, with the highest percentage of EDs over capacity in Massachusetts at $49 \%$ and the lowest in Colorado at $10 \%$. The exact determinants of this perceived variation are unknown, although population and provider density, along with health care resources, may play a role.

In terms of solutions implemented, the only high-impact solution that was implemented more in over capacity hospitals was the inpatient full capacity protocol. Overall, though, those hospitals whose EDs were operating over capacity had implemented a greater number of high-impact solutions than those with a more manageable census. Other solutions used more often by these hospitals included bedside registration/ elimination of triage, fast track units, and physicians at triage. Cancelling elective surgeries and smoothing the surgical schedule were most commonly (32\%) implemented at hospitals who defined their ED volume as a good balance and implemented slightly less commonly at those EDs that operate at and over capacity. However, beyond the scope of this study, 
Table 1 Emergency department characteristics by capacity

\begin{tabular}{|c|c|c|c|c|c|c|c|c|c|c|}
\hline \multicolumn{11}{|l|}{ Capacity } \\
\hline \multirow[t]{3}{*}{ Overall } & \multirow{3}{*}{$\begin{array}{l}\text { Total } \\
\mathrm{n} \\
284\end{array}$} & \multicolumn{2}{|c|}{ Under capacity } & \multicolumn{2}{|c|}{ Good balance } & \multicolumn{2}{|c|}{ At capacity } & \multicolumn{2}{|c|}{ Over capacity } & \multirow[t]{3}{*}{$\mathrm{P}$} \\
\hline & & $\mathrm{n}$ & $\%$ & $\mathrm{n}$ & $\%$ & $\mathrm{n}$ & $\%$ & $\mathrm{n}$ & $\%$ & \\
\hline & & 32 & $11 \%$ & 94 & $33 \%$ & 85 & $30 \%$ & 73 & $26 \%$ & \\
\hline \multicolumn{11}{|l|}{ State } \\
\hline Colorado & 62 & 10 & $16 \%$ & 33 & $53 \%$ & 13 & $21 \%$ & 6 & $10 \%$ & \multirow[t]{4}{*}{$<0.001$} \\
\hline Georgia & 115 & 9 & $8 \%$ & 41 & $36 \%$ & 34 & $30 \%$ & 31 & $27 \%$ & \\
\hline Massachusetts & 59 & 2 & $3 \%$ & 5 & $8 \%$ & 23 & $39 \%$ & 29 & $49 \%$ & \\
\hline Oregon & 48 & 11 & $23 \%$ & 15 & $31 \%$ & 15 & $31 \%$ & 7 & $15 \%$ & \\
\hline \multicolumn{11}{|l|}{ Urban influence } \\
\hline Urban & 159 & 13 & $8 \%$ & 48 & $30 \%$ & 54 & $34 \%$ & 44 & $28 \%$ & \multirow[t]{3}{*}{0.31} \\
\hline Rural, adjacent to urban & 70 & 11 & $16 \%$ & 24 & $34 \%$ & 19 & $27 \%$ & 16 & $23 \%$ & \\
\hline Rural, not adjacent to urban & 55 & 8 & $15 \%$ & 22 & $40 \%$ & 12 & $22 \%$ & 13 & $24 \%$ & \\
\hline \multicolumn{11}{|l|}{ Teaching hospital } \\
\hline No & 264 & 31 & $12 \%$ & 90 & $34 \%$ & 76 & $29 \%$ & 67 & $25 \%$ & \multirow[t]{2}{*}{0.35} \\
\hline Yes & 20 & 1 & $5 \%$ & 4 & $20 \%$ & 9 & $45 \%$ & 6 & $30 \%$ & \\
\hline \multicolumn{11}{|l|}{ Critical access hospital } \\
\hline No & 216 & 24 & $11 \%$ & 71 & $33 \%$ & 66 & $31 \%$ & 55 & $25 \%$ & \multirow[t]{2}{*}{0.98} \\
\hline Yes & 68 & 8 & $12 \%$ & 23 & $34 \%$ & 19 & $28 \%$ & 18 & $26 \%$ & \\
\hline \multicolumn{11}{|l|}{ Visit volume (patients/hour) } \\
\hline$<1$ & 59 & 11 & $19 \%$ & 40 & $68 \%$ & 8 & $14 \%$ & 0 & $0 \%$ & \multirow[t]{4}{*}{$<0.001$} \\
\hline $1-1.9$ & 60 & 4 & $7 \%$ & 28 & $47 \%$ & 23 & $38 \%$ & 5 & $8 \%$ & \\
\hline $2.0-2.9$ & 30 & 6 & $20 \%$ & 6 & $20 \%$ & 12 & $40 \%$ & 6 & $20 \%$ & \\
\hline$\geq 3$ & 135 & 11 & $8 \%$ & 20 & $15 \%$ & 42 & $31 \%$ & 62 & $46 \%$ & \\
\hline \multicolumn{11}{|l|}{ Admission rate } \\
\hline $0-10 \%$ & 66 & 7 & $11 \%$ & 27 & $41 \%$ & 22 & $33 \%$ & 10 & $15 \%$ & \multirow[t]{3}{*}{0.16} \\
\hline $11-20 \%$ & 156 & 16 & $10 \%$ & 51 & $33 \%$ & 43 & $28 \%$ & 46 & $29 \%$ & \\
\hline$>20 \%$ & 49 & 5 & $10 \%$ & 10 & $20 \%$ & 18 & $37 \%$ & 16 & $33 \%$ & \\
\hline \multicolumn{11}{|l|}{ Uninsured } \\
\hline $0-15 \%$ & 86 & 11 & $13 \%$ & 23 & $27 \%$ & 22 & $26 \%$ & 30 & $35 \%$ & \multirow[t]{3}{*}{0.08} \\
\hline $16-30 \%$ & 114 & 15 & $13 \%$ & 34 & $30 \%$ & 38 & $33 \%$ & 27 & $24 \%$ & \\
\hline$>30 \%$ & 59 & 4 & $7 \%$ & 28 & $47 \%$ & 14 & $24 \%$ & 13 & $22 \%$ & \\
\hline
\end{tabular}

the possibility that implementation of these surgical solutions improved ED volume at these hospitals merits further study. Location of an ED, whether by state, urban vs. rural, or critical access hospital did not have any impact on the composite score, nor did teaching hospital status or proportion of uninsured patients. Only visit volume (patients per hour) and admission proportion (\%) increased with an increased composite score. A greater proportion of admitted patients might impede patient flow, and this impediment, along with higher ED volume, appears to be a significant motivation for the implementation of crowding solutions in our study. When controlling for other variables in the multivariate model, only a visit volume that equaled or exceeded three patients/hour was found to be significantly associated with an increased composite score.

The movement of boarding patients out of the ED to inpatient hallways (the "full capacity protocol") was pioneered at SUNY Stony Brook, and has since been found to not only decrease the ED length of stay (LOS), but also inpatient LOS by 1 day [7], while resulting in no change in mortality rates between patients moved to inpatient hallways and those not in hallway beds [8].

The coordination of discharge for inpatients prior to noon has been proposed to improve the availability of inpatient beds, especially in the afternoon and evening periods. However,few data exist to support this notion. A simulation model based on real patient data suggested that discharging $75 \%$ of the inpatients by noon actually increased boarding hours, whereas a uniform discharge plan for all patients between noon and midnight, along with one that matches the timing of admissions to discharges, significantly decreased boarding hours [4].

Bedside registration has been found to decrease overall ED LOS by 10-15 min [9, 10]. A number of studies have also demonstrated the positive impact of fast track units on wait times, with improved throughput of low-acuity patients, reduced LWBS rates, and shorter overall ED LOS. However, these results have all been in single center studies, and multi-center investigations are needed to 
Table 2 Crowding solutions implemented by capacity

Capacity

\begin{tabular}{|c|c|c|c|c|c|c|c|c|c|c|}
\hline & \multirow{2}{*}{$\begin{array}{l}\text { Total } \\
\mathrm{n}\end{array}$} & \multicolumn{2}{|c|}{ Under capacity } & \multicolumn{2}{|c|}{ Good balance } & \multicolumn{2}{|c|}{ At capacity } & \multicolumn{2}{|c|}{ Over capacity } & \multirow[t]{2}{*}{$\mathrm{P}$} \\
\hline & & $\mathrm{n}$ & $\%$ & $\mathrm{n}$ & $\%$ & $\mathrm{n}$ & $\%$ & $\mathrm{n}$ & $\%$ & \\
\hline \multicolumn{11}{|l|}{ High-impact solutions } \\
\hline Inpatient full capacity protocols & 68 & 7 & $10 \%$ & 17 & $25 \%$ & 17 & $25 \%$ & 27 & $40 \%$ & 0.03 \\
\hline Inpatient discharge coordination & 196 & 21 & $11 \%$ & 66 & $34 \%$ & 53 & $27 \%$ & 56 & $29 \%$ & 0.21 \\
\hline Surgical Schedule Smoothing & 110 & 10 & $9 \%$ & 35 & $32 \%$ & 31 & $28 \%$ & 34 & $31 \%$ & 0.63 \\
\hline \multicolumn{11}{|l|}{ Additional solutions } \\
\hline Bedside registration/eliminating triage & 206 & 26 & $13 \%$ & 70 & $34 \%$ & 52 & $25 \%$ & 58 & $28 \%$ & 0.03 \\
\hline Fast track units & 144 & 12 & $8 \%$ & 23 & $16 \%$ & 46 & $32 \%$ & 63 & $44 \%$ & $<0.001$ \\
\hline Observations units & 69 & 4 & $6 \%$ & 26 & $38 \%$ & 17 & $25 \%$ & 22 & $32 \%$ & 0.16 \\
\hline Physician triage & 42 & 3 & $7 \%$ & 7 & $17 \%$ & 12 & $29 \%$ & 20 & $48 \%$ & 0.005 \\
\hline Cancelling elective surgeries & 68 & 10 & $15 \%$ & 22 & $32 \%$ & 16 & $24 \%$ & 20 & $29 \%$ & 0.45 \\
\hline \multicolumn{11}{|l|}{ Non-effective solutions } \\
\hline ED bed expansion & 108 & 9 & $8 \%$ & 33 & $31 \%$ & 31 & $29 \%$ & 35 & $32 \%$ & 0.20 \\
\hline \multicolumn{11}{|l|}{ Diverted patients } \\
\hline Left before being seen, median (IQR) & 266 & 26 & $1(1-2)$ & 87 & $1(1-3)$ & 81 & $2(1-3)$ & 72 & $3(2-4)$ & $<0.001$ \\
\hline Ambulance diversion hours/month, median (IQR) & 134 & 15 & $10(2-35)$ & 26 & $4(2-30)$ & 41 & $10(4-15)$ & 52 & $18(5-58)$ & 0.08 \\
\hline
\end{tabular}

produce generalizable results [11]. A physician at triage has had a variable impact on the throughput of patients [12-16].

Without the coordination of elective cases, a large amount of artificial variation is created in bed demand. One institution found that $70 \%$ of all ICU diversions were associated with peaks in the volume of elective surgical cases [17]. Similarly, the smoothing of elective medical admissions for treatments such as chemotherapy is necessary in order to control the demand for the fixed amount of hospital resources.

Interestingly, access to consultants, not crowding, is the primary safety concern for rural emergency physicians [18]. Nevertheless, the concept of crowding and its adverse effects on patient care have been well defined in the medical literature, leading to the ACEP report that recommended a number of crowding solutions [1]. However, as our study demonstrates, only a small percentage of EDs across the country have implemented these solutions.
ED crowding is a definite problem in the US health care system. However, based on our findings, it appears to be a problem for a certain percentage of EDs. These solutions may not be necessary for lower volume EDs whose resources may be better utilized in other ways to improve the care of their patients. As the area of ED crowding research evolves, our focus should be the right solutions targeted to the right environments instead of a general approach that will have a variable impact, depending on the capacity constraints of each ED.

\section{Limitations}

Given that these data were collected through surveys, they are subject to both recall and response bias. A large proportion of respondents were from urban areas, which may have skewed
Table 3 Univariable analysis of high-impact crowding solutions by capacity

\begin{tabular}{|c|c|c|c|c|c|c|c|c|c|}
\hline \multicolumn{10}{|l|}{ Capacity } \\
\hline \multirow[t]{2}{*}{ Number of high-impact solutions* } & \multirow{2}{*}{$\begin{array}{l}\text { Total } \\
\mathrm{n}\end{array}$} & \multicolumn{2}{|c|}{ Under capacity } & \multicolumn{2}{|c|}{ Good balance } & \multicolumn{2}{|c|}{ At capacity } & \multicolumn{2}{|c|}{ Over capacity } \\
\hline & & $\mathrm{n}$ & $\%$ & $\mathrm{n}$ & $\%$ & $\mathrm{n}$ & $\%$ & $\mathrm{n}$ & $\%$ \\
\hline 0 & 63 & 8 & $13 \%$ & 20 & $32 \%$ & 23 & $37 \%$ & 12 & $19 \%$ \\
\hline 1 & 81 & 11 & $14 \%$ & 29 & $36 \%$ & 25 & $31 \%$ & 16 & $20 \%$ \\
\hline 2 & 83 & 6 & $7 \%$ & 29 & $35 \%$ & 18 & $22 \%$ & 30 & $36 \%$ \\
\hline 3 & 37 & 4 & $11 \%$ & 9 & $24 \%$ & 11 & $30 \%$ & 13 & $35 \%$ \\
\hline
\end{tabular}


Table 4 Univariable analysis of composite number of any crowding solution of at and over capacity emergency departments

\begin{tabular}{|c|c|c|c|}
\hline & \multicolumn{3}{|c|}{ Composite score (out of 9) } \\
\hline & \multirow[t]{2}{*}{ Mean } & \multicolumn{2}{|c|}{$(95 \% \mathrm{CI})$} \\
\hline \multicolumn{3}{|l|}{ State } & \\
\hline Colorado & 4.6 & 3.7 & 5.5 \\
\hline Georgia & 3.9 & 3.3 & 4.4 \\
\hline Massachusetts & 3.6 & 3.0 & 4.2 \\
\hline Oregon & 4.2 & 3.5 & 4.9 \\
\hline \multicolumn{4}{|l|}{ Urban influence } \\
\hline Urban & 3.8 & 3.3 & 4.2 \\
\hline Rural, adjacent to urban & 4.1 & 3.3 & 4.8 \\
\hline Rural, not adjacent to urban & 4.3 & 3.4 & 5.1 \\
\hline \multicolumn{4}{|l|}{ Teaching hospital } \\
\hline No & 3.9 & 3.6 & 4.3 \\
\hline Yes & 3.8 & 2.5 & 5.1 \\
\hline \multicolumn{4}{|l|}{ Critical access hospital } \\
\hline No & 3.8 & 3.5 & 4.2 \\
\hline Yes & 4.1 & 3.5 & 4.8 \\
\hline \multicolumn{4}{|l|}{ Visit volume (patients/hour) } \\
\hline$<1$ & 1.2 & 0.6 & 1.8 \\
\hline $1-1.9$ & 2.4 & 1.6 & 3.2 \\
\hline $2.0-2.9$ & 3.4 & 2.4 & 4.4 \\
\hline$\geq 3$ & 4.6 & 4.2 & 4.9 \\
\hline \multicolumn{4}{|l|}{ Admission rate } \\
\hline $0-10 \%$ & 2.9 & 2.2 & 3.7 \\
\hline $11-20 \%$ & 4.0 & 3.6 & 4.5 \\
\hline$>20 \%$ & 4.6 & 3.9 & 5.2 \\
\hline \multicolumn{4}{|l|}{ Uninsured } \\
\hline $0-15 \%$ & 3.7 & 3.1 & 4.3 \\
\hline $16-30 \%$ & 4.2 & 3.6 & 4.7 \\
\hline$>30 \%$ & 3.8 & 2.9 & 4.6 \\
\hline
\end{tabular}

the generalizability of the results. There were no objective operational metrics obtained from EDs to confirm the accuracy of their survey responses. For example, capacity was subjectively determined by ED directors. However, this is the most common methodology available and has been used by the American Hospital Association in previous studies [6]. Given this history, most current policy discussions have used this definition. In addition, the ACEP report came out in April 2008 and may have influenced the implementation of some of these solutions in EDs included in the study population since the period of study was the calendar year 2008. Lastly, those that marked "Partial/ In Progress" were grouped with "Yes" responses for solutions implementation, thereby possibly over-counting the prevalence of such practices.
Table 5 Multivariable linear regression analysis of composite number of any crowding solution for at and over capacity EDs

\begin{tabular}{|c|c|c|c|c|}
\hline & $\beta$ & \multicolumn{2}{|c|}{$(95 \% \mathrm{CI})$} & $\mathrm{P}$ \\
\hline Colorado & 0.27 & -0.83 & 1.37 & 0.63 \\
\hline Georgia & \multicolumn{4}{|c|}{ Reference } \\
\hline Massachusetts & -0.84 & -1.73 & 0.04 & 0.06 \\
\hline Oregon & 0.12 & -1.04 & 1.29 & 0.83 \\
\hline \multicolumn{5}{|l|}{ Urban influence } \\
\hline Urban & \multicolumn{4}{|c|}{ Reference } \\
\hline Rural, adjacent to urban & 0.18 & -0.72 & 1.08 & 0.70 \\
\hline Rural, not adjacent to urban & 0.11 & -0.97 & 1.19 & 0.84 \\
\hline \multicolumn{5}{|l|}{ Teaching hospital } \\
\hline No & \multicolumn{4}{|c|}{ Reference } \\
\hline Yes & -0.02 & -1.16 & 1.13 & 0.98 \\
\hline \multicolumn{5}{|l|}{ Critical access hospital } \\
\hline No & \multicolumn{4}{|c|}{ Reference } \\
\hline Yes & -0.11 & -1.08 & 0.87 & 0.83 \\
\hline \multicolumn{5}{|l|}{ Visit volume (patients/hour) } \\
\hline$<1$ & \multicolumn{4}{|c|}{ Reference } \\
\hline $1-1.9$ & 1.41 & -0.50 & 3.32 & 0.15 \\
\hline $2.0-2.9$ & 2.55 & 0.61 & 4.49 & 0.01 \\
\hline$\geq 3$ & 3.65 & 1.96 & 5.33 & $<0.001$ \\
\hline \multicolumn{5}{|l|}{ Admission rate } \\
\hline $0-10 \%$ & \multicolumn{4}{|c|}{ Reference } \\
\hline $11-20 \%$ & 0.55 & -0.40 & 1.50 & 0.25 \\
\hline$>20 \%$ & 0.91 & -0.28 & 2.11 & 0.13 \\
\hline \multicolumn{5}{|l|}{ Uninsured } \\
\hline $0-15 \%$ & \multicolumn{4}{|c|}{ Reference } \\
\hline $16-30 \%$ & 0.53 & -0.28 & 1.35 & 0.20 \\
\hline$>30 \%$ & 0.15 & -0.88 & 1.18 & 0.78 \\
\hline
\end{tabular}

\section{Conclusions}

Only a minority of EDs in 2008 reported being over capacity in our survey. This variability in capacity was associated with a range in the implementation of crowding solutions. With the intent to optimize the delivery of emergency care, future efforts in the use of these solutions might focus on EDs at or over capacity where they can make the greatest impact. For those EDs that are under capacity, improving access to care (i.e., improving the availability of specialty consultants) may be a more appropriate focus of their emergency care optimization.

Conflicts of interest None. 
Appendix

\section{E. CROWDING}

E1. On a typical day at $6 \mathrm{pm}$ :

a. Are there any ED patients being cared for primarily in the hallway?

$\square$, Yes

Do No

b. Are there patients that "board" in the ED for $>2$ hours until an inpatient bed becomes available?
$\square$ Y Yes
$\square$ №

E2. While patients are boarding in the ED please indicate the physician of record:

$\begin{array}{ll}\square_{1} & \text { Inpatient attending } \\ \square_{2} & \text { ED attending } \\ \square_{3} & \text { Other (specify): }\end{array}$

E3. Approximate percent of ED patients that left before being seen (LBBS)

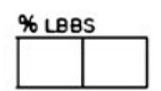

E4. In 2008, was your hospital ever on ambulance diversion? Di Yes $\quad \mathrm{Do}$ No (Go to E5)

If YES.

a. On average, over the entire year. approximately how many hours/month was your hosprtal on diversion?

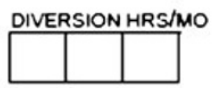

b Please indicate the number one reason for ambulance diversion in 2008 :

$\begin{array}{ll}\square_{1} & \text { ED staff shortages } \\ \square_{2} & \text { Lack of specialty physician coverage } \\ \square_{3} & \text { Lack of general acute care beds/staffing } \\ \square_{4} & \text { Lack of critical care beds/staffing } \\ \square_{5} & \text { Other (specify) }\end{array}$

E5. In 2008. how would you describe your hospital's ED?

$\square_{1} \quad$ Under capacity $\square_{3}$ At capacity

$\square_{2} \quad$ Good balance $\square_{4}$ Over capacity

E6. Has your hospital initiated the following:

\begin{tabular}{|c|c|c|c|c|}
\hline & & Yes & Progress & No \\
\hline a. & $\begin{array}{l}\text { Inpatient full capacity protocols- When the hospital is at capacity, your hospital moves admitted patients to } \\
\text { inpatient areas like hallways and other common areas. }\end{array}$ & $\square_{1}$ & $\square_{2}$ & 口o \\
\hline b. & $\begin{array}{l}\text { Inpatient discharge coordination- Your hosprtal makes concerted efforts to discharge patients before noon } \\
\text { through active management of services needed for the patient to leave the hospital such as dedicated case } \\
\text { managers and social workers }\end{array}$ & ום & $\square_{2}$ & 口o \\
\hline c. & $\begin{array}{l}\text { Suraical schedule smoothing- Instead of scheduling elective surgeries on certain days (eg. early in week). } \\
\text { your hospital works to spread out these cases over the entire week. }\end{array}$ & $\square_{1}$ & $\square_{2}$ & $\square 0$ \\
\hline d. & $\begin{array}{l}\text { Cancelling elective surgeries- When the hospital and/or ED is at capacity, your hospital administration } \\
\text { cancels scheduled elective surgical cases. }\end{array}$ & ום & $\square_{2}$ & 口o \\
\hline
\end{tabular}

E7. Has your ED initiated the following:

\begin{tabular}{|c|c|c|c|c|}
\hline & & Yes & Progress & No \\
\hline a. & $\begin{array}{l}\text { Bedside registration/Eliminating Triage- As long as an ED bed is available. patients are immediately brought } \\
\text { back from the waiting room upon amival, with registration happening at the bedside and eliminating triage. }\end{array}$ & $\square_{1}$ & $\square_{2}$ & 口o \\
\hline b. & Fast Track Unit- Patients with non-urgent medical conditions are sent to a separate area of the ED for care. & $\square 1$ & $\square 2$ & Do \\
\hline c. & $\begin{array}{l}\text { Observation Unit- A unit where patients can be admitted to treat simple medical conditions that would } \\
\text { otherwise warrant a short inpatient admission. }\end{array}$ & $\square 1$ & $\square_{2}$ & 口0 \\
\hline d. & $\begin{array}{l}\text { Physician Triage- A physician is specifically assigned to the trage area to help initiate work-ups on patients } \\
\text { and may discharge some low-acuity patients from triage without having to be brought back to a bed. }\end{array}$ & $\square 1$ & $\square_{2}$ & 口o \\
\hline e. & ED bed expansion- The number of beds in your ED increased over past 3 years. & $\square 1$ & $\square 2$ & $\square 0$ \\
\hline
\end{tabular}

Appendix: Crowding questions from survey

\section{References}

1. ACEP. Emergency Department Crowding: High-Impact Solutions. September 8, 2009. Available at: http://www.acep.org/WorkArea/ downloadasset.aspx ?id $=37960$.

2. GAO. Hospital Emergency Departments: Crowding Continues to Occur, and Some Patients Wait Longer than Recommended Time Frames. 2009.

3. Wilper AP, Woolhandler S, Lasser KE et al (2008) Waits to see an emergency department physician: US trends and predictors, 1997-2004. Health Aff (Millwood) 27(2):w8495
4. Powell E, Khare R, Venkatesh A, Van Roo B, Reinhardt G (2009) A hospital-wide approach to decreasing emergency department congestion: matching supply with demand. Acad Emerg Med 16(4):S146-S147

5. Sullivan AF, Richman IB, Ahn CJ et al (2006) A profile of US emergency departments in 2001. Ann Emerg Med 48(6):694-701

6. Emergency Department Overload: A Growing Crisis. The Results of the AHA Survey of Emergency Department (ED) and Hospital Capacity. The Lewin Group. Available at: http://www.aha.org/aha/ content $/ 2002 / \mathrm{pdf} /$ EdoCrisisSlides.pdf.

7. Innes G, Grafstein E, Stenstrom R, Harris D, Hunte G (2007) Impact of an overcapacity care protocol on emergency department overcrowding. Acad Emerg Med 14(5):S85

8. Viccellio A, Santora C, Singer AJ et al (2009) The association between transfer of emergency department boarders to inpatient hallways and mortality: a 4-year experience. Ann Emerg Med 54(4):487-491 
9. Gorelick MH, Yen K, Yun HJ (2005) The effect of in-room registration on emergency department length of stay. Ann Emerg Med 45(2):128133

10. Takakuwa KM, Shofer FS, Abbuhl SB (2007) Strategies for dealing with emergency department overcrowding: a 1-year study on how bedside registration affects patient throughput times. J Emerg Med 32(4):337-342

11. Wiler JL, Gentle C, Halfpenny JM et al (2010) Optimizing emergency department front-end operations. Ann Emerg Med $55: 142-160$

12. Crane P, Schneider S (2009) Effect of a triage doctor on length of stay in a crowded urban emergency department. Acad Emerg Med 16(4):S272

13. Gerton J, Schabelman E, Pimentel L, Ercolano P, Browne B, Barrueto B (2009) When is a physician in triage a financially viable option? Acad Emerg Med 16(4):S272

14. Holroyd BR, Bullard MJ, Latoszek K et al (2007) Impact of a triage liaison physician on emergency department overcrowding and throughput: a randomized controlled trial. Acad Emerg Med 14(8):702-708

15. Murrell K, Offerman S, Carl M, Kauffman M, Loeffler R (2009) Implementation of an emergency department physician in triage system decreases patient wait times and "left without being seen" rates. Acad Emerg Med 16(4):S204-S205

16. Partovi SN, Nelson BK, Bryan ED et al (2001) Faculty triage shortens emergency department length of stay. Acad Emerg Med 8 (10):990-995

17. McManus ML, Long MC, Cooper A et al (2003) Variability in surgical caseload and access to intensive care services. Anesthesiology 98(6):1491-1496

18. Sklar DP, Crandall CS, Zola T et al (2010) Emergency physician perceptions of patient safety risks. Ann Emerg Med 55(4):336-340
Daniel Handel, MD, MPH is the Director of Clinical Operations, Department of Emergency Medicine, Oregon Health \& Science University, Portland, Oregon, USA.

Dr. Adit Ginde is an Assistant Professor of Emergency Medicine at the University of Colorado School of Medicine. Dr. Ginde actively practices and teaches emergency medicine at University of Colorado Hospital and Denver Health Medical Center and is the Associate Director of the Colorado Emergency Medicine Research Center.

Ali S. Raja, MD, MBA is the Associate Director for Trauma, Department of Emergency Medicine, Brigham and Women's Hospital, Boston, Massachusetts, USA.

John Rogers, MD is an Emergency Physician in the Department of Emergency Medicine, Coliseum Northside Hospital, Macon, GA.

Ashley Sullivan, MS, MPH is the Associate Director of the Emergency Medicine Network, Department of Emergency Medicine, Massachusetts General Hospital, Boston, MA, USA.

Janice A. Espinola is the lead biostatistician/epidemiologist at the Emergency Medicine Network (EMNet, www.emnet-usa.org), responsible for data management and statistical analysis for all EMNet projects.

Carlos Camargo, MD, DrPH is an Emergency Physician at Massachusetts General Hospital and Associate Professor of Medicine \& Epidemiology at Harvard Medical School (Boston, MA) along with being the Principal Investigator of the Emergency Medicine Network (www.emnet-usa.org). 